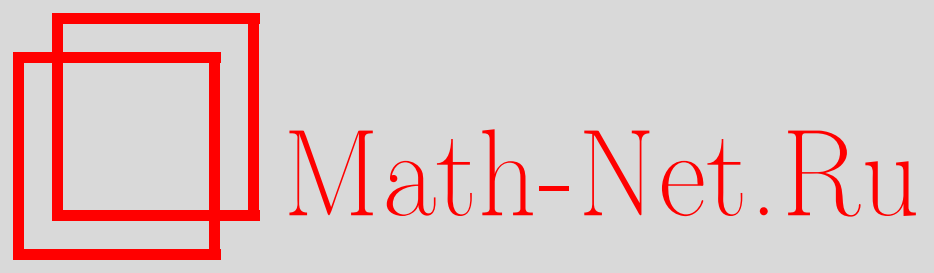

И. Антониадис, Е. Дудас, Д. М. Гиленча, П. Тзивелоглу, Нелинейная суперсимметрия и взаимодействия голдстино с минимальной суперсимметричной стандартной моделью, ТМФ, 2012, том 170, номер 1, 34-48

DOI: https://doi.org/10.4213/tmf6744

Использование Общероссийского математического портала Math-Net.Ru подразумевает, что вы прочитали и согласны с пользовательским соглашением http://www . mathnet.ru/rus/agreement

Параметры загрузки:

IP : 54.162 .127 .20

26 апреля 2023 г., 14:56:43

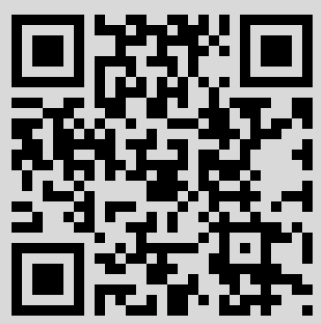



Д. М. Гиленча ${ }^{* \dagger \S}$, П. Тзивелоглу ${ }^{\dagger}$

\title{
НЕЛИНЕЙНАЯ СУПЕРСИММЕТРИЯ И ВЗАИМОДЕЙСТВИЯ ГОЛДСТИНО С МИНИМАЛЬНОЙ СУПЕРСИММЕТРИЧНОЙ СТАНДАРТНОЙ МОДЕЛЬЮ
}

\begin{abstract}
Приведен краткий обзор нелинейных суперсимметричных формализмов в стандартной реализации и суперполевых методов. Вычисляются взаимодействия голдстино с суперполями минимальной суперсимметричной Стандартной модели и обсуждаются их феноменологические следствия. Последние относятся к массе бозона Хиггса на древесном уровне и к невидимым распадам бозона Хиггса и $Z$-бозона. При низком масштабе нарушения суперсимметрии $\sqrt{f} \sim 2-7$ ТэВ масса бозона Хиггса увеличивается по сранению с ее значением на древесном уровне и поднимается выше уровня, задаваемого ограничением LEP2 по массе. Вычислены постоянные невидимого распада бозона Хиггса и $Z$-бозона в голдстино и нейтралино и показано, что при этом возникают более сильные ограничения на $f$, чем при их распадах на пары голдстино, которые относятся к следующему за ведущим порядку по $1 / f$.
\end{abstract}

Ключевые слова: суперсимметрия, Стандартная модель, нелинейная реализация симметрии.

\section{1. ВВЕДЕНИЕ}

Интерес к нелинейным реализациям суперсимметрии [1] восходит к ранним исследованиям суперсимметрии. Ниже мы приводим обзор формализма стандартной реализации (Волкова-Акулова) нелинейной суперсимметрии, а также суперполевое описание, которое было развито позже. В качестве применения суперполевого формализма со связями мы вычисляем все взаимодействия голдстино (которые преобразуются нелинейно при наличии суперсимметрии) с полями минимальной суперсимметричной Стандартной модели (MCCM) и исследуем ряд феноменологических следствий.

${ }^{*}$ Theory Division, CERN, Geneva, Switzerland. E-mail: Ignatios.AntoniadiS@cern.ch

${ }^{\dagger}$ Centre de Physique Théorique, Ecole Polytechnique, CNRS, Palaiseau, France

${ }^{\ddagger}$ Laboratoire de Physique Théorique, UMR du CNRS, Université de Paris-Sud, Paris, France

$\S$ Theoretical Physics Department, IFIN-HH Bucharest, Bucharest, Romania 
Спонтанное нарушение суперсимметрии при низких энергиях предсказывает практически безмассовое голдстино. Оно играет роль продольной компоненты гравитино, которое приобретает массу $f / M_{\text {Planck, }}$ в диапазоне милли-эВ, если масштаб нарушения суперсимметрии $\sqrt{f}$ находится в области мульти-ТэВ. Согласно теореме эквивалентности [2] оно взаимодействует с константой связи $1 / \sqrt{f}$, что гораздо сильнее, чем взаимодействия поперечного гравитино, подавленные по Планку, и поэтому хорошо описывается пределом безмассового голдстоуновского фермиона с отщепленной гравитацией. В настоящей работе обсуждаются низкоэнергетические следствия легкого голдстино в предположении, что $\sqrt{f}-$ независимый параметр, который на несколько порядков превышает члены, отвечающие мягкому нарушению в MCСМ. Ситуация следующая: при высоких энергиях (выше $\sqrt{f}$ ) имеется МССМ и суперполя голдстино. На ТэВ-масштабах (выше $m_{\text {soft }}$, но ниже $\sqrt{f}$ ) имеется МССМ вместе с "нелинейным" голдстино. При более низких энергиях, ниже масс суперпартнеров $\left(\sim m_{\mathrm{soft}}\right)$, остается только голдстиновский фермион, взаимодействующий с полями Стандартной модели (CM). Мы рассматриваем энергетическую область $E \sim m_{\text {soft }}<\sqrt{f}$, где эффективные взаимодействия голдстино определяются нелинейной суперсимметрией.

Самодействия голдстино задаются известным действием Волкова-Акулова [1]. С помощью этого метода, геометрического по своей природе, можно также получить универсальное взаимодействие голдстино с материей через тензор энергии-импульса, который имеет вид $\left(1 / f^{2}\right) T_{\mu \nu} t^{\mu \nu}$, где $T_{\mu \nu}$ и $t_{\mu \nu}$ - тензоры энергии-импульса материи и (свободного) голдстино соответственно [3], [4]. Однако было замечено, что это взаимодействие не является наиболее общим инвариантом относительно преобразований нелинейной суперсимметрии [4]-[6]. Общие инвариантные взаимодействия можно получить, используя так называемые суперполевые формулировки нелинейной суперсимметрии. Одна из них продвигает обычное поле до уровня суперполя путем введения модифицированного суперпространства с учетом нелинейных преобразований суперсимметрии голдстино [7]-[10]. Наконец, имеется метод суперполей со связями: это обычные суперполя, однако они подчинены связям, исключающим суперпартнеров путем выражения их в терминах легких степеней свободы и (вейлевского) голдстино [11]-[14].

Ниже мы приводим краткий обзор этих методов и представляем соответствующие взаимодействия голдстино, индуцированные заданным лагранжианом при "суперсимметризации" нелинейным способом. Далее мы приводим обзор метода суперполей со связями, а затем обсуждаем феноменологические следствия для МССМ, взаимодействующей с нелинейно преобразующимся полем голдстино. Эти феноменологические следствия относятся к: 1) поправке к массе бозона Хиггса, которую можно повысить уже на древесном уровне по сравнению с ее МССМ-значением на древесном уровне для скрытого сектора на суперсимметричном масштабе в области нескольких ТэВ; 2) невидимым распадам бозона Хиггса и $Z$-бозона. Действительно, в главном порядке по взаимодействию голдстино бозон Хиггса и $Z$-бозон могут распадаться на голдстино и нейтралино (следующая за наилегчайшей суперсимметричной частицей $(\mathrm{CHCЧ}))^{1)}$ с достаточно большой постоянной распада, если суперсимметричный масштаб скрытого сектора низок (и СНСЧ является достаточно легкой).

\footnotetext{
1) Голдстино является наилегчайшей суперсимметричной частицей (НСЧ).
} 
Из точно измеренной постоянной распада $Z$-бозона $\Gamma_{Z}$ можно установить границы масштаба нарушения суперсимметрии $\sqrt{f}$. Что касается распада бозона Хиггса, то частичная вероятность этого распада может стать сравнимой с постоянной распада в пару фотонов в МССМ. Более того, он преобладает над другими каналами распада такими, как распад $Z$-бозона на пару голдстино, который пропорционален $O\left(1 / f^{2}\right)$ и ранее рассматривался в литературе. Эти эффекты представлены в последнем разделе.

\section{2. НЕЛИНЕЙНАЯ СУПЕРСИММЕТРИЯ В СТАНДАРТНОЙ РЕАЛИЗАЦИИ}

Для вывода закона преобразования нелинейной суперсимметрии сначала рассмотрим суперсимметричное преобразование $x^{\mu^{\prime}}=x^{\mu}+i\left(\theta \sigma^{\mu} \bar{\xi}-\xi \sigma^{\mu} \bar{\theta}\right), \theta^{\prime}=\theta+\xi$ и $\bar{\theta}^{\prime}=\bar{\theta}+\bar{\xi}$, где спинорные индексы не указаны. Это преобразование индуцирует нелинейную реализацию на спинорах $\theta$, которую на самом деле можно обобщить на произвольные спинорные поля, в частности вейлевский голдстиновский спинор $\chi$, по аналогии с $\theta$ и $\chi$. Здесь $\theta=\kappa \chi$, где $\kappa$ вводится из размерных соображений. Указанная аналогия дает [10]

$$
\chi^{\prime}\left(x^{\prime}\right)=\chi(x)+\frac{1}{\kappa} \xi
$$

После разложения в ряд Тейлора вблизи $x$ находим преобразование поля вейлевского голдстино:

$$
\delta \chi=\frac{1}{\kappa} \xi_{\alpha}+\kappa \Lambda_{\xi}^{\mu} \partial_{\mu} \chi
$$

где $\Lambda_{\xi}^{\mu}=i\left(\chi \sigma^{\mu} \bar{\xi}-\xi \sigma^{\mu} \bar{\chi}\right)$. Аналогичное соотношение, эрмитово сопряженное приведенному выше, выполнено для $\bar{\chi}$. Здесь $\Lambda_{\xi}^{\mu}-$ зависящий от поля голдстино вектор трансляции, а $\xi_{\alpha}, \bar{\xi}^{\dot{\alpha}}-$ (грассмановы) параметры преобразования; $\kappa$-постоянная распада голдстино, имеющая размерность массы ${ }^{-2}$ и связанная с масштабом нарушения (в скрытом секторе) суперсимметрии $\sqrt{f}=\Lambda_{\text {susy }}$ :

$$
\kappa=\frac{1}{\sqrt{2} f}=\frac{1}{\sqrt{2} \Lambda_{\text {susy }}^{2}} .
$$

Мы хотим построить лагранжиан для эффективного низкоэнергетического описания голдстино и его взаимодействий с полями СМ. Сначала рассмотрим часть эффективного действия, которая содержит только самодействия голдстино. Она должна содержать стандартный кинетический член для вейлевского спинора с дополнительным слагаемым, необходимым для инвариантности действия относительно стандартной нелинейной реализации (2). Действие, удовлетворяющее этим критериям, было построено в работе [1], где определен "репер"

$$
E_{\mu}^{\nu}=\delta_{\mu}^{\nu}+i \kappa^{2}\left(\partial_{\mu} \chi \sigma^{\nu} \bar{\chi}-\chi \sigma^{\nu} \partial_{\mu} \bar{\chi}\right)
$$

Используя преобразование (2), можно показать, что

$$
\delta(\operatorname{det} E)=i \kappa \partial_{\mu}\left[\left(\chi \sigma^{\mu} \bar{\xi}-\xi \sigma^{\mu} \bar{\chi}\right) \operatorname{det} E\right] \equiv \kappa \partial_{\mu}\left(\Lambda_{\xi}^{\mu} \operatorname{det} E\right) \text {. }
$$


Тогда естественно строится лагранжиан Акулова-Волкова [1]

$$
\mathcal{L}_{\mathrm{AV}}=-\frac{1}{2 \kappa^{2}} \operatorname{det} E=-\frac{1}{2 \kappa^{2}}+\frac{i}{2}\left(\chi \sigma^{\mu} \partial_{\mu} \bar{\chi}-\partial_{\mu} \chi \sigma^{\mu} \bar{\chi}\right)+\cdots,
$$

так что действие, определяемое посредством $\mathcal{L}_{\mathrm{AV}}$, инвариантно. Первый член в правой части представляет собой космологическую постоянную, второй член - обычный кинетический член для голдстино; точки обозначают самодействия, пропорциональные второй и выше степеням $\kappa$ и задающие нелинейное суперсимметричное расширение кинетической энергии вейлевского спинора. На самом деле разложение обрывается вследствие антикоммутативности спинора голдстино.

Алгебру суперсимметрии можно также реализовать нелинейно на других (неголдстиновских) полях таких, как поля материи и калибровочные [3], [4]. Обозначим через $\phi_{i}$ некоторое поле общего вида, где $i$ - индекс в некотором представлении группы Лоренца или внутренней группы симметрии. Определим

$$
\delta \phi_{i}=\kappa \Lambda_{\xi}^{\mu} \partial_{\mu} \phi_{i}
$$

Обычно при этом говорят о стандартной реализации. Можно проверить, что это дает представление суперсимметрии. Уравнение (7) имеет тот же вид, что и преобразование голдстино (2), за исключением отсутствия первого слагаемого. Однако производные $\phi_{i}$ (напряженность поля $F_{\mu \nu}$ и калибровочно-ковариантные производные $\left.D_{\mu} \phi_{i}\right)$ не преобразуются ковариантно согласно стандартной реализации даже несмотря на то, что так преобразуется поле $\phi_{i}$. Этого можно избежать с помощью обобщения обычной калибровочно-ковариантной производной путем определения нелинейно реализованной суперсимметрично-ковариантной производной

$$
\mathcal{D}_{\mu} \phi_{i} \equiv\left(E^{-1}\right)_{\mu}^{\nu} D_{\nu} \phi_{i}
$$

где $\left(E^{-1}\right)_{\mu}^{\nu}$-матрица, обратная матрице (4). Если $\phi_{i}-$ поле, преобразующееся в стандартной реализации, то $\mathcal{D}_{\mu} \phi_{i}$ также изменяется согласно стандартной реализации, $\delta\left(\mathcal{D}_{\mu} \phi_{i}\right)=\kappa \Lambda_{\xi}^{\mu}\left(\mathcal{D}_{\mu} \phi_{i}\right)$. Аналогичную процедуру можно применить к напряженности поля:

$$
\mathcal{F}_{\mu \nu}^{a} \equiv\left(E^{-1}\right)_{\mu}^{\sigma}\left(E^{-1}\right)_{\nu}^{\rho} F_{\sigma \rho}^{a},
$$

где $F_{\mu \nu}^{a}$ - обычная напряженность поля. Поэтому $\delta\left(\mathcal{F}_{\mu \nu}^{a}\right)=\kappa \Lambda_{\xi}^{\rho} \partial_{\rho} \mathcal{F}_{\mu \nu}^{a}$ и $\mathcal{F}_{\mu \nu}^{a}$ преобразуется согласно стандартной реализации. Если разложить правую часть выражения (9) по степеням $\kappa$, то первое слагаемое будет иметь вид $F_{\mu \nu}^{a}$, а далее идут соответствующие взаимодействия с полем голдстино. Это позволяет построить инвариантное эффективное действие. Если в лагранжиане СМ

$$
\mathcal{L}_{\mathrm{SM}}=\mathcal{L}_{\mathrm{SM}}\left(\phi_{i}, D_{\mu} \phi_{i}, F_{\mu \nu}^{a}\right)
$$

заменить все переменные из $\mathcal{L}_{\mathrm{SM}}$ на их аналоги, $F_{\mu \nu}^{a} \rightarrow \mathcal{F}_{\mu \nu}^{a}$ и $D_{\mu} \rightarrow \mathcal{D}_{\mu}$, то получающийся лагранжиан сам преобразуется как поле в стандартной реализации:

$$
\delta\left(\mathcal{L}_{\mathrm{SM}}\left(\phi_{i}, \mathcal{D}_{\mu} \phi_{i}, \mathcal{F}_{\mu \nu}^{a}\right)\right)=\kappa \Lambda_{\xi}^{\sigma} \partial_{\sigma} \mathcal{L}_{\mathrm{SM}}\left(\phi_{i}, \mathcal{D}_{\mu} \phi_{i}, \mathcal{F}_{\mu \nu}^{a}\right) .
$$

Далее, умножая $\mathcal{L}_{\mathrm{SM}}$ на $\operatorname{det} E$, находим

$$
S_{\text {eff }}=\int d^{4} x \mathcal{L}_{\text {eff }}=\int d^{4} x \operatorname{det} E \mathcal{L}_{\mathrm{SM}}\left(\phi_{i}, \mathcal{D}_{\mu} \phi_{i}, \mathcal{F}_{\mu \nu}^{a}\right),
$$


что инвариантно относительно преобразования суперсимметрии, как видно из преобразования для $\operatorname{det} E(5)$. При разложении эффективного лагранжиана $\mathcal{L}_{\text {eff }}$ по степеням $\kappa$ низший ( $\kappa$-независимый) член является лагранжианом самой СМ. Кроме того, $\kappa$-зависимые члены необходимы, чтобы сделать действие суперсимметричным; они описывают соответствующие взаимодействия СМ-полей с голдстино:

$$
\mathcal{L}_{\mathrm{eff}}=\mathcal{L}_{\mathrm{SM}}\left(\phi_{i}, D_{\mu} \phi_{i}, F_{\mu \nu}^{a}\right)+\left(i \kappa^{2} \chi \grave{\partial}^{\mu} \sigma^{\nu} \bar{\chi}\right) T_{\mu \nu}+\cdots,
$$

где точки обозначают высшие степени $\kappa$ (которыми можно пренебречь в низкоэнергетическом пределе), а $T_{\mu \nu}$ - калибровочно-инвариантный тензор энергии-импульса.

Тензорное взаимодействие (13) полностью определено и является модельно-независимым. Однако описанная выше процедура построения взаимодействий голдстино не дает наиболее общего эффективного действия, инвариантного относительно нелинейной суперсимметрии. Дело в том, что к приведенному выше эффективному лагранжиану можно в принципе добавить дополнительные суперсимметричные члены. Эти члены, суперсимметричные сами по себе, могут входить в лагранжиан с произвольной относительной нормировкой, которую невозможно определить в рамках эффективной теории поля.

Можно применить два суперполевых формализма для нахождения общих взаимодействий голдстино с заданным лагранжианом. Первый из них продвигает всякое поле до суперполя путем введения модифицированного суперпространства, которое учитывает нелинейные суперсимметричные преобразования голдстино. Это сводится к сдвигу пространственно-временны́х координат $x^{\mu}$ на величину $\Lambda_{\theta}^{\mu}$ из выражения (2), где $\theta$ - обычные суперпространственные (вейлевские) координаты фермиона: $x^{\mu} \rightarrow \tilde{x}^{\mu}=x^{\mu}+\Lambda_{\theta}^{\mu}(\tilde{x})$. Аналогичная процедура применяется к вейлевскому фермиону голдстино, с которым ассоциируют суперполе голдстино: $\chi(x) \rightarrow \theta / \kappa+\chi(\tilde{x})$. $\mathrm{C}$ помощью этих объектов можно далее построить суперсимметричное действие, используя ковариантные производные, как ранее в терминах "репера" (4), зависящего от сдвинутых координат $\tilde{x}$. Подробности приведены в работах [7]-[10]. Второй формализм для нахождения общих взаимодействий голдстино с заданным лагранжианом - метод суперполей со связями.

\section{3. НЕЛИНЕЙНАЯ СУПЕРСИММЕТРИЯ С СУПЕРПОЛЯМИ СО СВЯЗЯМИ}

Очень удобный подход к нелинейной суперсимметрии заключается в использовании суперполей со связями. Этот метод сохраняет все преимущества работы в стандартном суперполевом формализме. В конечном итоге, роль этих связей заключается в том, чтобы исключить "массивные" суперпартнеры (для СМ и голдстино), выразив их в терминах легких степеней свободы (СМ-частиц и голдстино) [11]-[14] (см. также работы [15], [16]). Таким образом, получается нелинейная реализация суперсимметрии, в которой больше нет суперпартнеров. Как и в стандартной реализациии Волкова-Акулова, основную роль при построении нелинейной суперсимметричнной версии заданной модели играет киральное суперполе голдстино (СМ калибровочный синглет) $X$. Чтобы увидеть, как это работает, а также найти связь с $\mathcal{L}_{\mathrm{AV}}$ из формулы (6), рассмотрим лагранжиан [13], [14] (ниже $f$ связано с $\kappa$ 
как в формуле (3))

$$
\mathcal{L}_{X}=\int d^{4} \theta X^{\dagger} X-\left\{f \int d^{2} \theta X+\text { э.c. }\right\}=\left|\partial_{\mu} \varphi\right|^{2}+F^{\dagger} F+\left(\frac{i}{2} \bar{\chi} \bar{\sigma}^{\mu} \partial_{\mu} \chi-f F+\text { } \text {.c. }\right)
$$

и наложим связь на суперполе голдстино $X_{\mathrm{nl}}^{2}=0$. Эта связь решается в виде

$$
X_{\mathrm{nl}}=\varphi+\sqrt{2} \theta \chi+\theta \theta F
$$

где $\varphi=\chi \chi /(2 F)$, откуда ясно видно, как сголдстино $(\varphi)$ исключается, оказавшись выраженным в терминах голдстино $\chi$. Использование решения $X_{\mathrm{nl}}$ в $\mathcal{L}_{X}$ после исключения вспомогательных полей дает [13], [14]

$$
\mathcal{L}_{X}=-\frac{1}{2 \kappa^{2}}+i \bar{\chi} \bar{\sigma}^{\mu} \partial_{\mu} \chi+\frac{\kappa^{2}}{2} \bar{\chi}^{2} \partial^{2} \chi^{2}-\frac{\kappa^{6}}{2} \chi^{2} \bar{\chi}^{2} \partial^{2} \chi^{2} \partial^{2} \bar{\chi}^{2},
$$

что представляет собой как раз лагранжиан Волкова-Акулова $\mathcal{L}_{\mathrm{AV}}$; другими словами, $\mathcal{L}_{X}$ эквивалентен $\mathcal{L}_{\mathrm{AV}}$ на массовой оболочке (вне массовой оболочки это не так из-за наличия различных степеней свободы) [13]. Кроме того, на массовой оболочке $F=f+\cdots$, где $f$ - масштаб нарушения суперсимметрии скрытого сектора. При инфракрасном описании нарушения суперсимметрии скалярная компонента (сголдстино) $\varphi$ становится функцией голдстино $\chi$ и поэтому удаляется из спектра, который содержит только $\chi$, как в $\mathcal{L}_{\mathrm{AV}}$. Это имеет место для случая одного суперполя со связями - голдстино.

Могут иметься дополнительные связи, наложенные на материю и векторные суперполя, которые по существу получаются интегрированием некоторых суперпартнеров в терминах легких степеней свободы. Например,

$$
\begin{array}{rlrl}
Q_{\mathrm{nl}} X_{\mathrm{nl}} & =0 & & \text { исключает сфермионы, оставляет вейлевский фермион; } \\
\bar{D}^{\dot{\alpha}}\left[X_{\mathrm{nl}} H_{\mathrm{nl}}^{\dagger}\right] & =0 & & \text { исключает хиггсино, оставляет комплексный хиггс; } \\
X_{\mathrm{nl}} A_{\mathrm{nl}}-X_{\mathrm{nl}} A_{\mathrm{nl}}^{\dagger} & =0 & & \text { исключает фермион, оставляет вещественный скаляр; } \\
W_{\mathrm{nl}} X_{\mathrm{nl}} & =0 & \text { исключает гейджино, оставляет калибровочные поля. }
\end{array}
$$

Таким образом, добавляя к $\mathcal{L}$ все эти связи, получаем суперсимметричный лагранжиан, в котором все суперпартнеры "отынтегрированы" и отсутствуют в инфракрасном описании, будучи выражены в терминах легких степеней свободы. Последние единственные степени свободы, присутствующие в низкоэнергетическом спектре, и они обладают нелинейными правилами суперсимметричных преобразований. Однако не все из приведенных выше связей должны удовлетворяться одновременно. Например, в области энергий $m_{\text {soft }} \sim E \leqslant \sqrt{f}$ единственной связью является связь суперполя голдстино, подвергающегося нелинейному преобразованию, тогда как для случая МССМ его суперполя не имеют связей.

Следующий шаг заключается в том, чтобы найти взаимодействия голдстино с заданным лагранжианом таким, как лагранжиан МССМ. Стратегия состоит в том, чтобы записать эффективное разложение лагранжиана (функции всех суперполей, включая голдстино) по степеням $1 / f$ и ограничиться в нем заданным порядком. Этот метод является общим и удобным для нахождения взаимодействий, включающих более одного голдстино [14]. Будем следовать этой идее и рассмотрим суперсимметричную теорию с киральными мультиплетами $\Phi_{i} \equiv\left(\phi_{i}, \psi_{i}, F_{i}\right)$ и векторными 
мультиплетами $V \equiv\left(A_{\mu}^{a}, \lambda^{a}, D^{a}\right)$, которые наиболее общим образом взаимодействуют с $X_{\mathrm{nl}}$. Соответствующий лагранжиан имеет вид

$$
\begin{aligned}
\mathcal{L}=\int & d^{4} \theta\left[X_{\mathrm{nl}}^{\dagger} X_{\mathrm{nl}}+\Phi_{i}^{\dagger}\left(e^{V} \Phi\right)_{i}-\frac{m_{i}^{2}}{f^{2}} X_{\mathrm{nl}}^{\dagger} X_{\mathrm{nl}} \Phi_{i}^{\dagger}\left(e^{V} \Phi\right)_{i}\right]+\left\{\int d ^ { 2 } \theta \left[f X_{\mathrm{nl}}+W\left(\Phi_{i}\right)+\right.\right. \\
& \left.\left.+\frac{B_{i j}}{2 f} X_{\mathrm{nl}} \Phi_{i} \Phi_{j}+\frac{A_{i j k}}{6 f} X_{\mathrm{nl}} \Phi_{i} \Phi_{j} \Phi_{k}+\frac{1}{4}\left(1+\frac{2 m_{\lambda}}{f} X_{\mathrm{nl}}\right) \operatorname{Tr} W^{\alpha} W_{\alpha}\right]+ \text { э.c. }\right\}, \quad(17)
\end{aligned}
$$

где $m_{i}^{2}, B_{i j}, A_{i j k}$ - "мягкие" члены, а $m_{\lambda}$ - масса гейджино. Отсюда можно найти взаимодействия голдстино $(\chi)$ с обыкновенными материальными/калибровочными суперполями в главных порядках по $1 / f$.

Можно проверить, что эти взаимодействия эквивалентны взаимодействиям, полученным "стандартным путем" с помощью теоремы эквивалентности [2] из теории с соответствующим явным мягким нарушением (см. ниже), в которой голдстино взаимодействует следующим образом:

$$
\frac{1}{f} \partial^{\mu} \chi J_{\mu}=-\frac{1}{f} \chi \partial^{\mu} J_{\mu}+\text { (полная пространственно-временна́я производная). }
$$

Здесь $J_{\mu}$ - суперток теории, соответствующей выражению (17), в котором голдстино по существу заменяется на спурион $S$ согласно $S \equiv m_{\mathrm{soft}} \theta^{2} \leftrightarrow\left(m_{\mathrm{soft}} / f\right) X_{\mathrm{nl}}$ [14], где члены, соответствующие мягкому нарушению, имеют явный вид:

$$
\begin{aligned}
\mathcal{L}^{\prime}= & \int d^{4} \theta\left[1-m_{i}^{2} \theta^{2} \bar{\theta}^{2}\right] \Phi_{i}^{\dagger}\left(e^{V} \Phi\right)_{i}+\int d^{2} \theta\left[W\left(\Phi_{i}\right)-\frac{1}{2} B_{i j} \theta^{2} \Phi_{i} \Phi_{j}-\frac{1}{6} A_{i j k} \theta^{2} \Phi_{i} \Phi_{j} \Phi_{k}+\right. \\
& \left.+\frac{1}{4}\left(1-2 m_{\lambda} \theta^{2}\right) \operatorname{Tr} W^{\alpha} W_{\alpha}\right]+ \text { э.с. }
\end{aligned}
$$

С учетом этого уравнение (18) показывает, что на массовой оболочке все взаимодействия голдстино пропорциональны "мягким" членам. Действительно, суперток, отвечающий формуле $(19)$, и его дивергенция выражаются как $\left(\mathcal{D}_{\mu, i j}=\delta_{i j} \partial_{\mu}+i g A_{\mu}^{a} T_{i j}^{a}\right)$

$$
\begin{gathered}
J_{\alpha}^{\mu}=-\left[\sigma^{\nu} \bar{\sigma}^{\mu} \psi_{i}\right]_{\alpha}\left[\mathcal{D}_{\nu, i j} \phi_{j}\right]^{\dagger}+i\left[\sigma^{\mu} \bar{\psi}_{i}\right]_{\alpha} F_{i}-\frac{1}{2 \sqrt{2}}\left[\sigma^{\nu} \bar{\sigma}^{\rho} \sigma^{\mu} \bar{\lambda}^{a}\right]_{\alpha} F_{\nu \rho}^{a}+\frac{i}{\sqrt{2}} D^{a}\left[\sigma^{\mu} \bar{\lambda}^{a}\right]_{\alpha}, \\
\partial_{\mu} J_{\alpha}^{\mu}=\psi_{i, \alpha}\left(m_{i}^{2} \phi_{j}^{\dagger}+B_{i j} \phi_{j}+\frac{1}{2} A_{i j k} \phi_{j} \phi_{k}\right)+\frac{m_{\lambda}}{\sqrt{2}}\left[\left(\sigma^{\mu \nu}\right)_{\alpha}^{\beta} \lambda_{\beta}^{a} F_{\mu \nu}^{a}+D^{a} \lambda_{\alpha}^{a}\right]
\end{gathered}
$$

так что все взаимодействия голдстино пропорциональны "мягким" членам. Из уравнений (18), (20) можно воспроизвести взаимодействия с одним голдстино. Однако формализм суперполей со связями в формуле (17) обладает по сравнению с этой "стандартной процедурой" тем преимуществом, что его можно применить даже при вычислении взаимодействий с более чем одним голдстино. Как было упомянуто, это проделывается путем записи всех эффективных операторов (содержащих $X_{\mathrm{nl}}$ ) в некотором фиксированном порядке по $1 / f$. Сложнее найти их из уравнения (19). Однако следует отметить, что этот метод не учитывает возможные взаимодействия голдстино с материей, содержащие производные, которые могут появиться из тока Феррары-Зумино. 


\section{4. ВЗАИМОДЕЙСТВИЯ ГОЛДСТИНО С ПОЛЯМИ МССМ И СЛЕДСТВИЯ ИЗ НИХ}

Ниже мы исследуем некоторые феноменологические применения описанного выше формализма. Мы налагаем только связи на суперполе голдстино, т. е. это единственное поле с нелинейным преобразованием суперсимметрии в области энергии $E \sim m_{\mathrm{soft}}<\sqrt{f}$. При энергиях ниже $m_{\text {soft }}$ связи, аналогичные таковым для суперполя голдстино, должны применяться к самим суперполям МССМ, отвечающим интегрированию по соответствующим суперпартнерам, выражающему последние в терминах легких степеней свободы. Тем не менее задачи, которые мы решаем, на древесном уровне не затрагиваются дополнительными связями, наложенными на суперполя МССМ такие, как кварковые и лептонные суперполя. В действительности их можно наложить на полученные результаты на последующем этапе. Относящиеся сюда применения взаимодействий голдстино и феноменологии описаны также в работах [17]-[22].

\section{1. Эффективный лагранжиан взаимодействий голдстино с суперпо-} лями МССМ. Используя метод суперполей со связями, определим общие взаимодействия голдстино с суперполями MCCM, включающие один и два спинора голдстино. Как уже упоминалось, мы не учитываем взаимодействия голдстино с материей, включающие производные, которые в принципе могут возникать. Единственное отличие от обыкновенной MCСМ здесь в секторе, нарушающем суперсимметрию. Суперсимметрия нарушается спонтанно за счет вакуумного среднего $F$, которое фиксируется его уравнением движения (см. ниже). Тогда лагранжиан МССМ, взаимодействующей с голдстино, имеет вид

$$
\begin{aligned}
\mathcal{L}_{\mathrm{H}}= & \sum_{i=1,2} c_{i} \int d^{4} \theta X_{\mathrm{nl}}^{\dagger} X_{\mathrm{nl}} H_{i}^{\dagger} e^{V_{i}} H_{i}+\sum_{\Phi} c_{\Phi} \int d^{4} \theta X_{\mathrm{nl}}^{\dagger} X_{\mathrm{nl}} \Phi^{\dagger} e^{V} \Phi+\frac{B}{f} \int d^{2} \theta X_{\mathrm{nl}} H_{1} H_{2}+ \\
& +\frac{A_{u}}{f} \int d^{2} \theta X_{\mathrm{nl}} H_{2} Q U^{c}+\frac{A_{d}}{f} \int d^{2} \theta X_{\mathrm{nl}} Q D^{c} H_{1}+\frac{A_{e}}{f} \int d^{2} \theta X_{\mathrm{nl}} L E^{c} H_{1}+\text { э.c. }+ \\
& +\sum_{i=1}^{3} \frac{1}{16 g_{i}^{2} \zeta} \frac{2 m_{\lambda_{i}}}{f} \int d^{2} \theta X_{\mathrm{nl}} \operatorname{Tr}\left[W^{\alpha} W_{\alpha}\right]_{i}+\text { э.c. }
\end{aligned}
$$

где предполагается эффективное разложение по $1 / f$. В формуле $(21) c_{\Phi}=-m_{\Phi}^{2} / f^{2}$, где $\Phi \in\left\{Q, U^{c}, D^{c}, L, E^{c}\right\} ; m_{\Phi}$ - "мягкие" массы, которые можно выбрать равными $m_{0} ; \zeta$ сокращает следовый множитель. После исключения вспомогательных полей получаем новые взаимодействия $\mathcal{L}^{\text {new }}$ помимо обычных взаимодействий, отвечающих суперсимметричой части МССМ на массовой оболочке. Лагранжиан на массовой оболочке имеет вид

$$
\mathcal{L}^{\text {new }} \equiv \mathcal{L}_{F(1)}^{\text {aux }}+\mathcal{L}_{F(2)}^{\text {aux }}+\mathcal{L}_{D}^{\text {aux }}+\mathcal{L}_{m}^{\text {extra }}+\mathcal{L}_{g}^{\text {extra }}
$$

Два первых члена получаются путем исключения вспомогательных полей $F ; \mathcal{L}_{F(1)}^{\text {aux }}$ воспроизводит все "мягкие" члены из MCCM (не показаны) плюс космологический 
член; для $\mathcal{L}_{F(2)}^{\mathrm{aux}}$ с точностью до $O\left(1 / f^{3}\right)$ имеем

$$
\begin{aligned}
\mathcal{L}_{F(2)}^{\text {aux }}= & \left\{\frac { \overline { \chi } \overline { \chi } } { 2 f ^ { 2 } } \left[\mu\left(m_{1}^{2}+m_{2}^{2}\right) h_{1} \cdot h_{2}-\left(m_{1}^{2}+m_{Q}^{2}+m_{D}^{2}\right) h_{1} \cdot \phi_{Q} \phi_{D}-\right.\right. \\
& -\left(m_{1}^{2}+m_{L}^{2}+m_{E}^{2}\right) h_{1} \cdot \phi_{L} \phi_{E}-\left(m_{2}^{2}+m_{Q}^{2}+m_{U}^{2}\right) \phi_{Q} \phi_{U} \cdot h_{2}+ \\
& +\left(B h_{2}-A_{d} \phi_{Q} \phi_{D}-A_{e} \phi_{L} \phi_{E}\right)^{\dagger}\left(\mu h_{2}-\phi_{Q} \phi_{D}-\phi_{L} \phi_{E}\right)+ \\
& +\left(B h_{1}-A_{u} \phi_{Q} \phi_{U}\right)^{\dagger}\left(\mu h_{1}-\phi_{Q} \phi_{U}\right)+\left(A_{d} \phi_{D} h_{1}-A_{u} h_{2} \phi_{U}\right)^{\dagger}\left(\phi_{D} h_{1}-h_{2} \phi_{U}\right)+ \\
& \left.\left.+A_{d}\left(\left|\phi_{Q} \cdot h_{1}\right|^{2}+\left|\phi_{E} h_{1}\right|^{2}\right)+A_{u}\left|h_{2} \cdot \phi_{Q}\right|^{2}+A_{e}\left|\phi_{L} \cdot h_{1}\right|^{2}\right]+ \text { э.c. }\right\}- \\
& -\frac{1}{f^{2}} \mid B h_{1} \cdot h_{2}+A_{u} h_{2} \cdot \phi_{Q} \phi_{U}+A_{d} \phi_{Q} \phi_{D} \cdot h_{1}+A_{e} \phi_{L} \phi_{E} \cdot h_{1}+ \\
& +\frac{m_{\lambda_{i}}}{2} \lambda_{i} \lambda_{i}+\left.\left(m_{1}^{2}\left|h_{1}\right|^{2}+m_{2}^{2}\left|h_{2}\right|^{2}+m_{\Phi}^{2}\left|\phi_{\Phi}\right|^{2}\right)\right|^{2}- \\
& -\frac{1}{f}\left[m_{1}^{2} \bar{\chi} \bar{\psi}_{h_{1}} h_{1}+m_{2}^{2} \bar{\chi} \bar{\psi}_{h_{2}} h_{2}+m_{\Phi}^{2} \bar{\chi} \bar{\psi}_{\Phi} \phi_{\Phi}+\text { э.c. }\right]+O\left(\frac{1}{f^{3}}\right) .
\end{aligned}
$$

Это выражение содержит взаимодействия вейлевского голдстино с полями МССМ, но также и не зависящие от $\chi$ взаимодействия такие, как хиггсовы взаимодействия четвертого порядка. Суммирование понимается как суммирование по групповым индексам $\mathrm{CM}, i=1,2,3$, в гейджинном члене и по $\Phi=Q, U^{c}, D^{c}, L, E^{c}$ в массовых членах; соответствующие свертки между $S U(2)_{L}$-дублетами понимаются для голоморфных произведений, когда оказывается существенным указанный порядок. В последней строке имеются главные взаимодействия $O(1 / f)$, которые в действительности имеют размерность четыре по полям. Аналогичные взаимодействия существуют в $O\left(1 / f^{2}\right)$ и включают скалярные поля и поля гейджино. Наконец, матрицы Юкавы получаются из выражения (23) заменой $\phi_{Q} \phi_{D} \rightarrow \phi_{Q} \gamma_{d} \phi_{D}, \phi_{Q} \phi_{U} \rightarrow \phi_{Q} \gamma_{u} \phi_{U}$, $\phi_{L} \phi_{E} \rightarrow \phi_{L} \gamma_{e} \phi_{E}$

Имеются также новые взаимодействия для членов, включающих вспомогательные компоненты векторных суперполей СМ. Исключая их, находим

$$
\mathcal{L}_{D}^{\mathrm{aux}}=-\frac{1}{2} \sum_{i=1}^{3}\left[\widetilde{D}_{i}^{a}+\frac{1}{4 f^{2}}\left(m_{\lambda_{i}} \chi \chi+\text { э.с. }\right) \widetilde{D}_{i}^{a}+\frac{1}{\sqrt{2} f}\left(m_{\lambda_{i}} \chi \lambda_{i}^{a}+\text { э.c. }\right)\right]^{2}+O\left(\frac{1}{f^{3}}\right) .
$$

Это выражение дает взаимодействия гейджино-голдстино и хиггс-голдстино; $\widetilde{D}_{i}^{a}-$ вспомогательные поля МССМ:

$$
\begin{aligned}
& \widetilde{D}_{1}=-\frac{1}{2} g_{1}\left(-h_{1}^{\dagger} h_{1}+h_{2}^{\dagger} h_{2}+\frac{1}{3} \phi_{Q}^{\dagger} \phi_{Q}-\frac{4}{3} \phi_{U}^{\dagger} \phi_{U}+\frac{2}{3} \phi_{D}^{\dagger} \phi_{D}-\phi_{L}^{\dagger} \phi_{L}+2 \phi_{E}^{\dagger} \phi_{E}\right), \\
& \widetilde{D}_{2}^{a}=-\frac{1}{2} g_{2}\left(h_{1}^{\dagger} \sigma^{a} h_{1}+h_{2}^{\dagger} \sigma^{a} h_{2}+\phi_{Q}^{\dagger} \sigma^{a} \phi_{Q}+\phi_{L}^{\dagger} \sigma^{a} \phi_{L}\right), \\
& \widetilde{D}_{3}^{a}=-\frac{1}{2} g_{3}\left(\phi_{Q}^{\dagger} t^{a} \phi_{Q}-\phi_{U}^{\dagger} t^{a} \phi_{U}-\phi_{D}^{\dagger} t^{a} \phi_{D}\right) .
\end{aligned}
$$

Далее, полный лагранжиан содержит также новые взаимодействия, не индуцированные вспомогательными полями и отсутствущими в МССМ. В секторе материи 
они имеют вид

$$
\begin{aligned}
\mathcal{L}_{m}^{\text {extra }} & =\frac{1}{4 f^{2}}\left|\partial_{\mu}(\chi \chi)\right|^{2}+\left(\frac{i}{2} \bar{\chi} \bar{\sigma}^{\mu} \partial_{\mu} \chi+\text { э.c. }\right)- \\
& -\sum_{i=1}^{2} \frac{m_{i}^{2}}{f^{2}}\left\{\bar{\chi} \bar{\psi}_{h_{i}} \chi \psi_{h_{i}}+\left[\frac{i}{2}\left(\bar{\chi} \bar{\sigma}^{\mu} \chi\right)\left(h_{i}^{\dagger} \mathcal{D}_{\mu} h_{i}\right)+\frac{i}{2}\left|h_{i}\right|^{2} \bar{\chi} \bar{\sigma}^{\mu} \partial_{\mu} \chi+\ni . c .\right]\right\}- \\
& -\left[m_{i}^{2} \rightarrow m_{\Phi}^{2}, H_{i} \rightarrow \Phi\right]+\left\{\frac{B}{f}\left[\frac{1}{2 f} \chi \chi \psi_{h_{1}} \cdot \psi_{h_{2}}-h_{1} \cdot\left(\chi \psi_{h_{2}}\right)-\left(\chi \psi_{h_{1}}\right) \cdot h_{2}\right]+\right. \\
& +\frac{A_{u}}{f}\left[\frac{1}{2 f} \chi \chi\left(h_{2} \cdot \psi_{Q} \psi_{U}+\psi_{h_{2}} \cdot \phi_{Q} \psi_{U}+\psi_{h_{2}} \cdot \psi_{Q} \phi_{U}\right)-\chi\left(h_{2} \cdot \phi_{Q} \psi_{U}+h_{2} . \psi_{Q} \phi_{U}+\right.\right. \\
& \left.\left.+\psi_{h_{2}} \cdot \phi_{Q} \phi_{U}\right)\right]+\left[\frac { A _ { d } } { f } \left(\frac{1}{2 f} \chi \chi\left(\psi_{Q} \psi_{D} \cdot h_{1}+\phi_{Q} \psi_{D} \cdot \psi_{h_{1}}+\psi_{Q} \phi_{D} \cdot \psi_{h_{1}}\right)-\right.\right. \\
& \left.-\chi\left(\phi_{Q} \psi_{D} \cdot h_{1}+\psi_{Q} \phi_{D} \cdot h_{1}+\phi_{Q} \phi_{D} \cdot \psi_{h_{1}}\right)\right)+ \\
& +(D \rightarrow E, L \rightarrow Q)]+\ni . c .\}+O\left(\frac{1}{f^{3}}\right) .
\end{aligned}
$$

Отметим присутствие взаимодействий размерности 4 в таких полях, как $(B / f) h_{1} \chi \psi_{h_{2}}$, существенных для феноменологии при низких $f$. Наконец, имеются еще и новые взаимодействия в калибровочном секторе:

$$
\begin{aligned}
\mathcal{L}_{g}^{\text {extra }}= & \sum_{i=1}^{3} \frac{m_{\lambda_{i}}}{2 f}\left[\frac{\chi \chi}{-2 f}\left(2 i \lambda^{a} \sigma^{\mu} \Delta_{\mu} \bar{\lambda}^{a}-\frac{1}{2} F_{\mu \nu}^{a} F^{a \mu \nu}-\frac{i}{4} \epsilon^{\mu \nu \rho \sigma} F_{\mu \nu}^{a} F_{\rho \sigma}^{a}\right)-\right. \\
& \left.-\sqrt{2} \chi \sigma^{\mu \nu} \lambda^{a} F_{\mu \nu}^{a}\right]_{i}+\text { э.c. }+O\left(\frac{1}{f^{3}}\right),
\end{aligned}
$$

где $i=1,2,3$ - индекс калибровочной группы, а $\sigma^{\mu \nu}=(i / 4)\left(\sigma^{\mu} \bar{\sigma}^{\nu}-\sigma^{\nu} \bar{\sigma}^{\mu}\right)$.

$\mathcal{L}^{\text {new }}$ вместе с частью на массовой оболочке чисто суперсимметричной части лагранжиана МССМ дает окончательное выражение для действия, содержащего все взаимодействия голдстино с МССМ с одним и двумя голдстино. Некоторые из феноменологических следствий отсюда рассмотрены ниже.

4.2. МССМ-потенциал Хиггса и поправки к массам. Из выражения для $\mathcal{L}^{\text {new }}$ извлекается полный скалярный потенциал. Для сектора Хиггса он имеет вид

$$
\begin{aligned}
V=f^{2} & +\left(|\mu|^{2}+m_{1}^{2}\right)\left|h_{1}\right|^{2}+\left(|\mu|^{2}+m_{2}^{2}\right)\left|h_{2}\right|^{2}+\left(B h_{1} \cdot h_{2}+\text { э.c. }\right)+ \\
& +\left.\frac{1}{f^{2}}\left|m_{1}^{2}\right| h_{1}\right|^{2}+m_{2}^{2}\left|h_{2}\right|^{2}+\left.B h_{1} \cdot h_{2}\right|^{2}+\frac{g_{1}^{2}+g_{2}^{2}}{8}\left[\left|h_{1}\right|^{2}-\left|h_{2}\right|^{2}\right]^{2}+ \\
& +\frac{g_{2}^{2}}{2}\left|h_{1}^{\dagger} h_{2}\right|^{2}+O\left(\frac{1}{f^{3}}\right) .
\end{aligned}
$$

Отметим первый член в последней строке, порождаемый на массовой оболочке, имеющий размерность 4 по полям и не представленный в МССМ. Его происхождение в конечном счете обусловлено связью на суперполе голдстино и исключением сголдстино в терминах легких степеней свободы (вейлевское голдстино). При больших $f$ и фиксированных "мягких" массах воспроизводится обычный МССМ-предел. Этот 
скалярный потенциал дает поправки к значению массы бозона Хиггса в МССМ. Их точный вид можно найти в работе [21]; при больших $u \equiv \operatorname{tg} \beta$ и фиксированном $m_{A}$ находим

$$
\begin{aligned}
& m_{h}^{2}=\left[m_{Z}^{2}+O\left(\frac{1}{u^{2}}\right)\right]+\frac{v^{2}}{2 f^{2}}\left[\left(2 \mu^{2}+m_{Z}^{2}\right)^{2}+O\left(\frac{1}{u^{2}}\right)\right]+O\left(\frac{1}{f^{3}}\right), \\
& m_{H}^{2}=\left[m_{A}^{2}+O\left(\frac{1}{u^{2}}\right)\right]+\frac{1}{f^{2}} O\left(\frac{1}{u^{2}}\right)+O\left(\frac{1}{f^{3}}\right) .
\end{aligned}
$$

Поэтому $m_{h}$ увеличивается по сравнению со своим значением в МССМ на древесном уровне. Это увеличение определяется большими $\mu$ и, по-видимому, имеет суперсимметричное происхождение, однако взаимодействия Хиггса четвертого порядка, обусловливающие этот эффект, включают комбинации "мягких" масс (см. формулу (28)). Эти мягкие массы комбинируются таким образом, чтобы на электрослабом масштабе дать зависящее от $\mu$ увеличение в выражениях (29). Как видно из рис. 1, имеется разумная область в пространстве параметров, в которой масса Хиггса может достигать значения LEP2,

равного 114.4 ГэВ, даже на древесном уровне из-за эффективных поправок, связанных с интегрированием по скалярам и вспомогательным полям для суперполя голдстино. Этот результат важен в силу того, что он не требует новой физики в видимом секторе для разрешения несоответствия между ограничением в МССМ на древесном уровне и ограничением LEP2. Это происходит в случае достаточно низкого масштаба нарушения суперсимметрии, $\sqrt{f} \sim 2 \div 7$ ТэВ. Данный эффект мог бы также оказаться существенным для сценария, в котором масса Хиггса определяется как масса зведомо выше границы 114.4 ГэВ ${ }^{2)}$. В этом случае МССМ требует больших квантовых поправок (и немалого количества тонкой настройки электрослабого масштаба).

4.3. Распады невидимых бозонов Хиггса и $Z$. Из взаимодействий поля голдстино с полями МССМ вытекают некоторые интересные следствия. Одно из них состоит в том, что для СНСЧ нейтралино имеется возможность распада нейтральных Хиггсов в голдстино и СНСЧ $\chi_{1}^{0}$. Взаимодействие хиггс-голдстино-нейтралино подавлено только фактором $1 / f$. Он возникает из следующих членов в $\mathcal{L}^{\text {new }}$ и из членов в суперсимметричной части обычного лагранжиана MCCM, далее обозначаемого как $\mathcal{L}_{0}^{\text {onshell }}$

$$
\begin{aligned}
\mathcal{L}^{\text {new }}+\mathcal{L}_{0}^{\text {onshell }} \supset & -\frac{1}{f}\left[m_{1}^{2} \chi \psi_{h_{1}^{0}} h_{1}^{0 *}+m_{2}^{2} \chi \psi_{h_{2}^{0}} h_{2}^{0 *}\right]-\frac{B}{f}\left[\chi \psi_{h_{2}^{0}} h_{1}^{0}+\chi \psi_{h_{1}^{0}} h_{2}^{0}\right]- \\
& -\frac{1}{f} \sum_{i=1,2} \frac{m_{\lambda_{i}}}{\sqrt{2}} \widetilde{D}_{i}^{a} \chi \lambda_{i}^{a}-\frac{1}{\sqrt{2}}\left[g_{2} \lambda_{2}^{3}-g_{1} \lambda_{1}\right]\left[h_{1}^{0 *} \psi_{h_{1}^{0}}-h_{2}^{0 *} \psi_{h_{2}^{0}}\right]+\text { э.c. }
\end{aligned}
$$

Если СНСЧ является достаточно легкой, $m_{\chi_{1}^{0}}<m_{h}$, то $h^{0}, H^{0}$ могут распадаться на нее плюс голдстино, которое имеет массу порядка $f / M_{\text {Planck }} \sim 10^{-3}$ эВ $^{3)}$.

${ }^{2)} \mathrm{B}$ МССМ масштаб тонкой электрослабой настройки минимизируется при $m_{h} \approx 114 \pm 2$ ГэВ, а за пределами этого значения он возрастает экспоненциально до уровня $1 / 1000$ при $m_{h} \approx$ 122 ГэВ [23] (см. также работу [24]).

3) Если это не так, то имеет место распад нейтралино на $h^{0}$ и голдстино, что рассматривалось в работе [25]. 


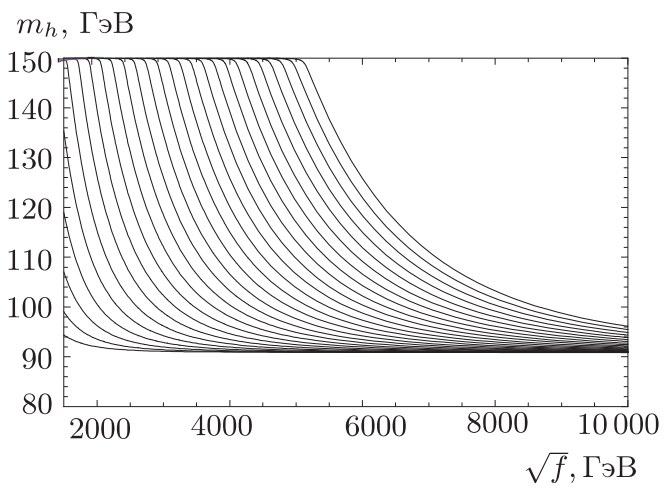

a

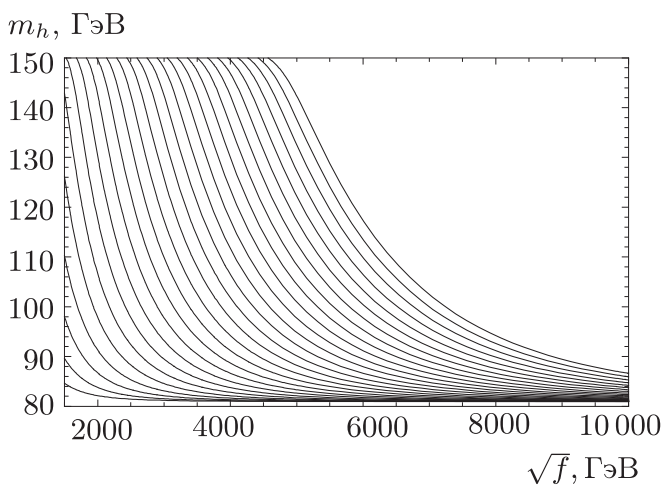

б

Рис. 1. Хиггсовы массы на древесном уровне как функции $\sqrt{f} ; m_{A}=$ 150 ГэВ и $m_{h}$ возрастает при изменении $\mu$ от 400 до 3000 ГэВ с шагом 100 ГэВ; $\mathrm{a}-\operatorname{tg} \beta=50, \sigma-\operatorname{tg} \beta=5$. Зависимость от $\operatorname{tg} \beta$ слабее, чем в МССМ.

Постоянная распада равна

$$
\Gamma_{h^{0} \rightarrow \chi_{1}^{0} \chi}=\frac{m_{h}}{16 \pi f^{2}}\left|\sum_{k=1}^{4} \delta_{k}^{\prime} \mathcal{Z}_{1 k}\right|^{2}\left(1-\frac{m_{\chi_{1}^{0}}^{2}}{m_{h^{0}}^{2}}\right)^{2},
$$

где $\mathcal{Z}$ - матрица, диагонализующая массовую матрицу нейтралино. Частичная вероятность распада получает поправки от полей и хиггсино $\left(\mathcal{Z}_{13}, \mathcal{Z}_{14}\right)$, и гейджино $\left(\mathcal{Z}_{11}, \mathcal{Z}_{12}\right)$, поскольку и то, и другое приобретают компоненту голдстино. Гейджиновская поправка возникает после смешивания гейджино-голдстино и нарушения суперсимметрии и электрослабой симметрии (это видно из зависимости $m_{\lambda_{i}}$ и $m_{Z}$ от $\delta_{k}^{\prime}$; последняя дается в работе [21]) и ранее не учитывалась в аналогичных исследованиях [22], [25], [26].

На рис. 2 частичная вероятность распада представлена для различных значений $\mu, m_{A}$ и $m_{\lambda_{1,2}}$. Большая постоянная распада требует легкого $\mu \sim O(100)$ ГэВ, когда нейтралино $\chi_{1}^{0}$ обладет хиггсиновой компонентой. В то же время возрастание $m_{h}$ выше ограничения LEP требует большего значения $\mu$, близкого к $\mu \approx 700$ ГэВ, если $\sqrt{f} \approx 1.5$ ТэВ, и $\mu \approx 850$ ГэВ, если $\sqrt{f} \approx 2$ ТэВ (см. рис. 1а). Результаты, представленные на рис. 2, показывают, что частичная вероятность распада может быть существенной $\left(\sim 3 \cdot 10^{-6}\right.$ ГэВ), если вспомнить, что полная постоянная распада Хиггса в СМ (при $m_{h} \approx 114$ ГэВ) составляет около $3 \cdot 10^{-3}$ ГэВ, причем вероятность распада $h^{0} \rightarrow \gamma \gamma$ равна $2 \cdot 10^{-3}$ (см. рис. 2 в работе [27]). Таким образом, вероятность распада по данному процессу может быть близкой к таковой для $h^{0} \rightarrow \gamma \gamma$ в СМ. Распад не очень чувствителен к $\operatorname{tg} \beta$ (рис. 2б) из-за дополнительного (не относящегося к MCCM) вклада от взаимодействия Хиггса четвертого порядка. Интересно было бы проанализировать указанную постоянную распада на однопетлевом уровне, чтобы провести более тщательное сравнение с постоянными распадов Хиггса в СМ.

Другое взаимодействие, также присутствующее в главном порядке $(1 / f)$ - взаимодействие голдстино с $Z_{\mu}$-бозоном и с нейтралино. В зависимости от отношения масс оно может провести к распаду $Z_{\mu}\left(\chi_{j}^{0}\right)$ в $\chi_{j}^{0}\left(Z_{\mu}\right)$ и в голдстино соответственно. 


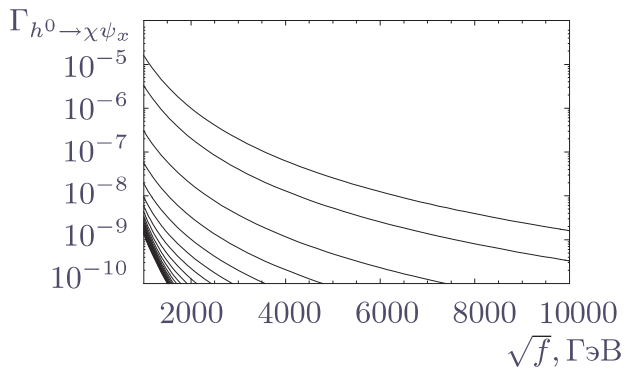

a

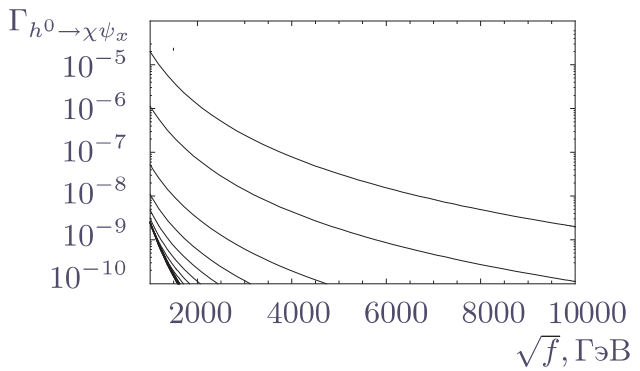

б

Рис. 2. Частичная вероятность распада $h^{0} \rightarrow \chi \chi_{1}^{0}$ при: $\mathrm{a}-\operatorname{tg} \beta=50$, $m_{\lambda_{1}}=70$ ГэВ, $m_{\lambda_{2}}=150$ ГэВ, $\mu$ возрастает от 50 ГэВ (верхняя кривая) с шагом 50 ГэВ,$m_{A}=150$ ГэВ (ср. с рис. 1a, соответствующим сходному диапазону параметров; при бо́льших $\mu, m_{h}$ возрастает, но частичная вероятность распада уменьшается); б $-\operatorname{tg} \beta=5$ (ср. с рис. 1б). Полная постоянная распада в СМ при $m_{h} \sim 114$ ГэВ имеет порядок $10^{-3}$, и вероятность распада в указанных случаях становится сравнимой с таковой для хиггса из СM, превращающегося в $\gamma \gamma$ (см. рис. 2 из работы [27]).

При этом существенные члены имеют вид

$$
\begin{aligned}
\mathcal{L}^{\text {new }}+\mathcal{L}_{0}^{\text {onshell }} \supset & -\frac{1}{4} \bar{\psi}_{h_{1}^{0}} \bar{\sigma}^{\mu} \psi_{h_{1}^{0}}\left(g_{2} V_{2}^{3}-g_{1} V_{1}\right)_{\mu}+\frac{1}{4} \bar{\psi}_{h_{2}^{0}} \bar{\sigma}^{\mu} \psi_{h_{2}^{0}}\left(g_{2} V_{2}^{3}-g_{1} V_{1}\right)_{\mu}- \\
& -\sum_{i=1}^{2} \frac{m_{\lambda_{i}}}{\sqrt{2} f} \chi \sigma^{\mu \nu} \lambda_{i}^{a} F_{\mu \nu, i}^{a}+\text { э.c. },
\end{aligned}
$$

где последний член обусловлен формулой (27) ( $i$ нумерует калибровочную группу). Поскольку хиггсино приобретают голдстиновскую компоненту $(\propto \chi / f)$ посредством массового смешивания, из первой приведенной выше строчки индуцируются дополнительные $O(1 / f)$-взаимодействия хиггсино с голдстино и с $Z_{\mu}=$ $(1 / g)\left(g_{2} V_{2}^{3}-g_{1} V_{1}\right)_{\mu}$, причем $g^{2}=g_{1}^{2}+g_{2}^{2}$. Если при этом $m_{\chi_{1}^{0}}$ легче, чем $Z_{\mu}$, то становится возможным распад последнего в $\chi_{1}^{0}+\chi$. Постоянная распада по этому процессу имеет вид (при $j=1$ )

$$
\Gamma_{Z \rightarrow \chi \chi_{j}^{0}}=\frac{m_{Z}^{5}}{32 \pi f^{2}}\left[\zeta_{1}\left|w_{j}\right|^{2}+\zeta_{2}\left|v_{j}\right|^{2}+\zeta_{3}\left(w_{j} v_{j}^{*}+w_{j}^{*} v_{j}\right)\right]\left(1-\frac{m_{\chi_{j}}^{2}}{m_{Z}^{2}}\right)^{2},
$$

где $\zeta_{1}=2\left(2+r^{2}\right) \mu^{2} / m_{Z}^{2}, \zeta_{2}=2\left(8+r^{2}\right)\left(1+2 r^{2}\right), \zeta_{3}=-2\left(4+5 r^{2}\right) \mu / m_{Z}$ при $r=m_{\chi_{j}} / m_{Z}$. Эта постоянная распада должна находиться в пределах ошибки LEP для $\Gamma_{Z}$, равной 2.3 МэВ [28] (если игнорировать теоретические неопределенности, которые малы). Отсюда получается нижняя граница для $\sqrt{f}$, которая может оказаться на уровне $\sqrt{f} \approx 700$ ГэВ для рассмотренного на рис. 1 пространства параметров, тогда как значения общего положения равны $\sqrt{f} \sim O(400)$ ГэВ. Поэтому результаты, касающиеся возрастания $m_{h}$, где требовалось значение $\sqrt{f}$ в ТэВ-й области, не имеют этого ограничения. Это ограничение неприменимо, если наилегчайшее нейтралино имеет массу, бо́льшую $m_{Z}$ (это можно устроить, например, за счет большей $m_{\lambda_{1}}$ ), когда имеет место противоположный распад $\left(\chi_{j} \rightarrow Z \chi\right)$. 
Есть также интересная возможность невидимого распада калибровочного $Z_{\mu}$-бозона в пару полей голдстино, который мы рассматривали в работах [6], [14], [18]. Он обусловлен следующими членами лагранжиана (после того, как хиггсовское поле приобретает вакуумное среднее):

$$
\begin{aligned}
\mathcal{L}^{\text {new }} & +\mathcal{L}_{0}^{\text {onshell }} \supset\left\{\frac{1}{4 f^{2}} \bar{\chi} \bar{\sigma}^{\mu} \chi\left(g_{2} V_{2}^{3}-g_{1} V_{1}\right)_{\mu}\left(\frac{m_{1}^{2} v_{1}^{2}}{2}-\frac{m_{2}^{2} v_{2}^{2}}{2}\right)-\right. \\
& \left.-\frac{1}{4} \bar{\psi}_{h_{1}^{0}} \bar{\sigma}^{\mu} \psi_{h_{1}^{0}}\left(g_{2} V_{2}^{3}-g_{1} V_{1}\right)_{\mu}+\frac{1}{4} \bar{\psi}_{h_{2}^{0}} \bar{\sigma}^{\mu} \psi_{h_{2}^{0}}\left(g_{2} V_{2}^{3}-g_{1} V_{1}\right)_{\mu}\right\}+ \text { э.c. }
\end{aligned}
$$

Тогда постоянная распада равна

$$
\Gamma_{Z \rightarrow \chi \chi}=\frac{m_{Z}}{24 \pi g^{2}}\left[\frac{m_{Z}^{4}}{2 f^{2}}\right]^{2} \cos ^{2}(2 \beta),
$$

что согласуется с предыдущими результатами, полученными для $B=0$ [6], [14], [18]. Постоянная распада не зависит от $m_{A}$ и должна находиться в пределах ошибки LEP для $\Gamma_{Z}(2.3 \mathrm{MэB} \mathrm{[28]).} \mathrm{Тогда} \mathrm{легко} \mathrm{видеть,} \mathrm{что} \mathrm{возрастание} \mathrm{массы} \mathrm{бозона} \mathrm{Хиггса}$ за пределы ограничения LEP (114.4 ГэВ), которое мы видим на рис. 1, согласуется с текущими ограничениями этой постоянной распада, что налагает на $f$ лишь "мягкие" связи при энергиях ниже ТэВ-х ( 200 ГэВ) [6], [18]. Причина этого в дополнительных подавляющих степенях $1 / f$ по сравнению с постоянными распада, вычисленными ранее в данном разделе. Аналогичный анализ можно провести и для распада наилегчайших бозонов Хиггса в пару голдстино, однако при этом мы не получим никаких существенных ограничений для $f$ из-за сильного подавления $\left(\sim 1 / f^{4}\right)$.

\section{5. ЗАКЛЮЧЕНИЕ}

Мы привели краткий обзор нелинейной суперсимметрии в стандартной реализации Волкова-Акулова и в формализме суперполей со связями. Последний из перечисленных методов нетрудно приложить к МССМ для вычисления взаимодействий суперполей МССМ с голдстиновским фермионом. Это возможно для области энергий выше $m_{\text {soft }}$ и ниже масштаба нарушения суперсимметрими $\sqrt{f}$, который предполагается в несколько раз выше, чем "мягкие" массы. В этой области энергий единственное поле, претерпевающее нелинейные преобразования, - поле голдстино. Используя эту идею, мы вычислили все взаимодействия голдстино с МССМ до порядка $O\left(1 / f^{2}\right)$ включительно. Что касается феноменологии, то интересным следствием является возрастание массы наилегчайшего бозона Хиггса по сравнению с ее значением в МССМ на древесном уровне, которое приближается к ограничению LEP2 при $\sqrt{f} \sim 2 \div 7$ ТэB. Это значение согласуется с имеющимися ограничениями, происходящими из невидимых распадов $Z$-бозона и бозона Хиггса. Наконец, важно подчеркнуть основные отличия предложенной модели от МССМ: наличие дополнительной легкой фермионной моды, а именно голдстино (или, эквивалентно, продольного гравитино), являющейся СМ-синглетом, и существование дополнительной симметрии, а именно нелинейной суперсимметрии. 
Благодарности. Настоящая работа выполнена при частичной финансовой поддержке Европейской комиссии (контракты PITN-GA-2009-237920 и ERC Advanced Grant 226371 ("MassTeV")), INTAS (грант 03-51-6346), ANR (CNRS-USAR) (контракт 05-BLAN-007901), CNRS PICS 3747 и 4172, а также Romanian National Authority for Scientific Research (CNCS UEFISCDI проект PN-II-ID-PCE-2011-3-0607).

\section{Список литературы}

[1] D. V. Volkov, V. P. Akulov, Phys. Lett. B, 46:1 (1973), 109-110.

[2] P. Fayet, Phys. Lett. B, 70:4 (1977), 461-464; 84:4 (1979), 421-426; 86:3-4 (1979), 272-278; R. Casalbuoni, S. De Curtis, D. Dominici, F. Feruglio, R. Gatto, Phys. Lett. B, 215:2 (1988), 313-316; Phys. Rev. D, 39:8 (1989), 2281-2288.

[3] T. E. Clark, S. T. Love, Phys. Rev. D, 54:9 (1996), 5723-5727, arXiv: hep-ph/9608243.

[4] T.E. Clark, T. Lee, S. T. Love, G. Wu, Phys. Rev. D, 57:9 (1998), 5912-5915, arXiv: hep-ph/9712353.

[5] A. Brignole, F. Feruglio, F. Zwirner, JHEP, 11 (1997), 001, 11 pp. arXiv: hep-th/9709111.

[6] M. A. Luty, E. Pontón, Phys. Rev. D, 57:7 (1998), 4167-4173, arXiv: hep-ph/9706268.

[7] E. A. Ivanov, A. A. Kapustnikov, J. Phys. A, 11:12 (1978), 2375-2384; Erratum, 12:2 (1979), 259; J. Phys. G, 8:2 (1982), 167-191.

[8] S. Samuel, J. Wess, Nucl. Phys. B, 221:1 (1983), 153-177.

[9] I. Antoniadis, M. Tuckmantel, Nucl. Phys. B, 697:1-2 (2004), 3-47, arXiv: hep-th/0406010.

[10] J. Wess, J. Bagger, Supersymmetry and Supergravity, Princeton Univ. Press, Princeton, NJ, 1992.

[11] M. Roček, Phys. Rev. Lett., 41:7 (1978), 451-453.

[12] U. Lindström, M. Roček, Phys. Rev. D, 19:8 (1979), 2300-2303.

[13] R. Casalbuoni, S. De Curtis, D. Dominici, F. Feruglio, R. Gatto, Phys. Lett. B, 220:4 (1989), 569-575.

[14] Z. Komargodski, N. Seiberg, JHEP, 09 (2009), 066, 34 pp., arXiv: 0907.2441.

[15] A. A. Zheltukhin, On equivalence of the Komargodski-Seiberg action to the Volkov-Akulov action, arXiv: 1009.2166.

[16] S. M. Kuzenko, S. J. Tyler, Phys. Lett. B, 698:4 (2011), 319-322, arXiv: 1009.3298.

[17] I. Antoniadis, M. Tuckmantel, F. Zwirner, Nucl. Phys. B, 707:1-2 (2005), 215-232, arXiv: hep-ph/0410165.

[18] A. Brignole, J. A. Casas, J. R. Espinosa, I. Navarro, Nucl. Phys. B, 666:1-2 (2003), 105-143, arXiv: hep-ph/0301121.

[19] L. Álvarez-Gaumé, C. Gómez, R. Jimenez, Phys. Lett. B, 690:7 (2010), 68-72, arXiv: 1001.0010.

[20] F. Luo, K. A. Olive, M. Peloso, The gravitino coupling to broken gauge theories applied to the MSSM, arXiv: 1006.5570.

[21] I. Antoniadis, E. Dudas, D. M. Ghilencea, P. Tziveloglou, Nucl. Phys. B, 841:1-2 (2010), 157-177, arXiv: 1006.1662.

[22] A. Djouadi, M. Drees, Phys. Lett. B, 407:3-4 (1997), 243-249, arXiv: hep-ph/9703452.

[23] S. Cassel, D. M. Ghilencea, G. G. Ross, Nucl. Phys. B, 835:1-2 (2010), 110-134, arXiv: 1001.3884 .

[24] S. Cassel, D. M. Ghilencea, G. G. Ross, Nucl. Phys. B, 825:1-2 (2010), 203-221, arXiv: 0903.1115 .

[25] S. Dimopoulos, S. Thomas, J. D. Wells, Nucl. Phys. B, 488:1-2 (1997), 39-91, arXiv: hep-ph/9609434.

[26] S. Ambrosanio, G. L. Kane, G. D. Kribs, S. P. Martin, S. Mrenna, Phys. Rev. D, 54:9 (1996), 5395-5411, arXiv: hep-ph/9605398.

[27] A. Djouadi, J. Kalinowski, M. Spira, Comput. Phys. Commun., 108:1 (1998), 56-74, arXiv: hep-ph/9704448.

[28] C. Amsler et al. (Particle Data Group), Phys. Lett. B, 667:1-5 (2008), 1-6. 